\title{
Effect of ramp-like contours \\ upon perceived size and detection threshold'
}

\author{
J. P. THOMAS 2 AND L. KERR \\ UNIVERSITY OF CALIFORNIA, LOS ANGELES
}

\begin{abstract}
Perceived widths and detection thresholds were measured for foveally viewed stimuli which were edged on two sides by luminance ramps. Perceived width approximated the distance between the midpoints of the ramps. It varied with the width of the ramps, but was unaffected by a five-fold change in the luminance of the stimulus. The detection threshold was higher for a ramp stimulus than for a normal stimulus of the same apparent width. The relationship between perceived width and detectability is consistent with a theoretical treatment based upon receptive-field mechanisms.
\end{abstract}

Charman (1963) studied the perceived dimensions of disks of light by having the $\mathrm{O}$ locate the apparent edges with a crosshair. The disks were presented in a microscope and, because of diffraction, their physical edges were gradual changes in intensity rather than abrupt steps. In every case, the perceived edge lay well within the region of changing intensity, part way up the intensity gradient. One possible explanation of this finding is that the edge is perceived where the intensity gradient first exceeds the difference threshold with respect to the background. Another type of explanation is possible, however, that involves spatial interactions and their effects either upon the distribution of sensation magnitudes (von Békésy, 1960) or upon the determination of which of several receptive-field mechanisms responds to the stimulus (Thomas, 1968b; Thomas, Rourke, \& Wilder, 1968). The threshold explanation is perhaps more parsimonious, but the interaction hypotheses have the advantage in that they can also account for the changes in brightness and detectability that accompany changes in the sharpness of edge-gradients (Enoch, 1958; Thomas \& Kovar, 1965). In the present study, the perceived widths of foveally viewed stimuli with ramp-like edges were measured by a size-matching procedure. The luminances of the ramp stimuli were varied in order to select between the threshold and interaction explanations. Detection thresholds were also obtained because the analysis advanced by Thomas (1968b) entails certain relationships between perceived width and detectability.

\section{Stimuli}

\section{METHOD AND PROCEDURE}

The stimuli were rectangles of incandescent light. All were $40 \mathrm{~min}$ of visual angle high. The comparison stimuli and some of the test stimuli were bounded on all sides by luminance steps. An example of such step stimuli is on the left side of Fig. 1. The remaining test stimuli were bounded laterally by luminance ramps as shown on the right side of Fig. 1. In such a ramp stimulus, the luminance along any vertical was constant over the height of the stimulus and terminated in steps at the top and bottom. The luminance varied along any horizontal as shown by the luminance plot at the bottom. The width of the central plateau, $W_{1}$, was $10 \mathrm{~min}$ for all ramp stimuli. The overall width, $W_{2}$, varied from one stimulus to another. The luminance of ramp stimuli was measured at the center. The solid and broken lines show the effect of varying luminance. All stimuli were superimposed on a square background, $15 \mathrm{deg}$ on a side, which was continuously illuminated at $40 \mathrm{~mL}$. In order to guide S's accommodation and fixation, a square area, $2 \mathrm{deg}$ on a side, was outlined by fine, dark lines. All stimuli
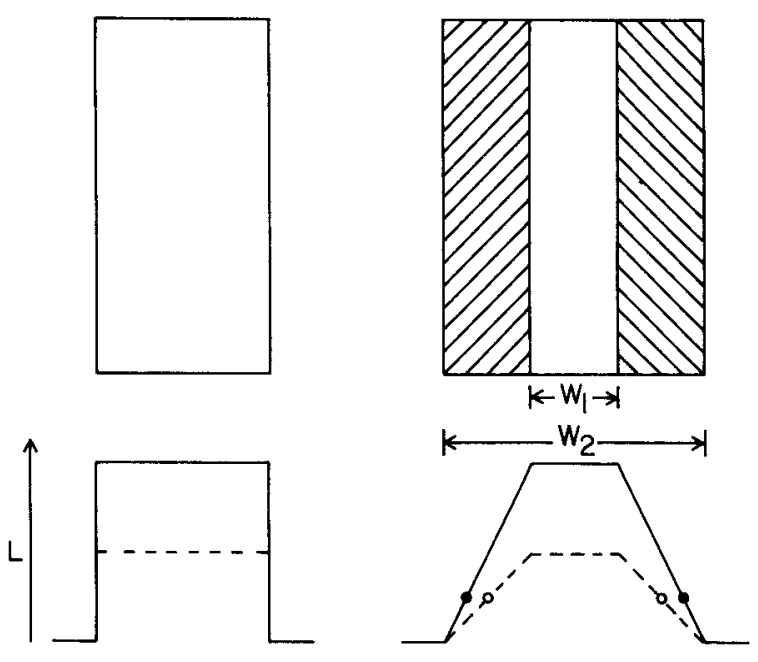

Fig. 1. Step stimulus, at left, and ramp stimulus, at right. The bottom figures show the variation in luminance $(L)$ across the width of each stimulus at two luminance levels.

were presented in the center of this area. $S$ was instructed to fixate the center of the area. The optical viewing distance was $2 \mathrm{~m}$ for all stimuli.

\section{Apparatus}

The stimuli were generated with a cylindrical lens after the manner described by Thomas (1967). Details of the present apparatus appear in Fig. 2. Light from a tungsten filament lamp, $S_{1}$, was focused on a small aperture where its passage was controlled by Shutter Sh, which was driven by a Brush pen motor. Opening the shutter illuminated a small disk of opal glass, OG. Lens $L_{2}$ imaged this patch on the aperture at $B$, uniformly illuminating the aperture. Each point on the lens projected uniformly to all points of the aperture. Thus, the lens served as the source for the remainder of the system. The aperture for the test stimulus was at A, next to the lens. The apertures for the step stimuli were rectangles with vertical sides and the openings for the ramp stimuli were trapezoids with sloping sides. Cylindrical lens $L_{c}$ formed a onedimensional image, $1 \mathrm{~mm}$ high, of the stimulus aperture at the artificial pupil, AP. The aperture at B determined the height of the stimuli. Neutral density filters at $F$ controlled the luminance of the stimuli. A duplicate pathway presented the comparison stimuli. The background was a sheet of opal glass, $\mathrm{Bg}$, illuminated by tungsten lamp $\mathrm{S}_{2}$. The fixation lines were etched on a sheet of glass at Fx, which was placed at the same optical distance as the stimuli by Spherical Lens $\mathrm{L}_{3}$. The artificial pupil, AP, was $3 \mathrm{~mm}$ in diam.

Absolute luminance calibrations were made with a Spectra Brightness Spot Meter placed at the artificial pupil and aligned with the optical axis of the system. The Spectra was calibrated with its entrance pupil stopped down to $3 \mathrm{~mm}$, the size of the artificial pupil. Thus, a stimulus was assigned a given luminance if the stimulus produced the same retinal illuminance as a diffusely reflecting surface of the same 


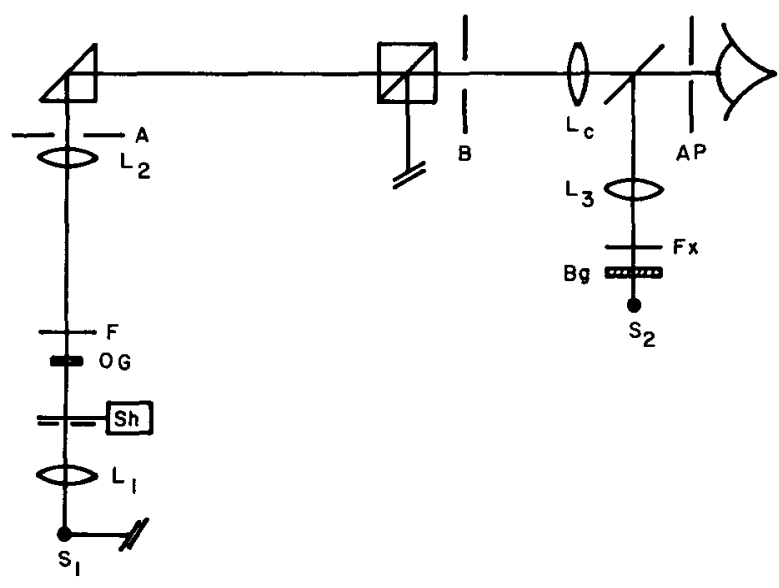

Fig. 2. Apparatus used to generate the stimuli.

luminance viewed through a $3-\mathrm{mm}$ pupil. The relative luminance distributions within the various stimuli were verified by placing a $55-\mathrm{mm}$ lens at the artificial pupil and scanning the resulting images with a Gamma 700-10-25 scanning micrometer eyepiece ( 50 micron probe) and associated photometer.

\section{EXPERIMENT 1}

The perceived widths of step and ramp stimuli were measured at three luminance levels. If the apparent width of a ramp stimulus corresponds to the portion of the stimulus which exceeds the difference threshold, then a given stimulus should appear wider at a high luminance than at a low one. (Let the circles in Fig. 1 be at threshold and compare the distance between the closed circles with the distance between the open circles.) The step stimuli were included to provide a reference for the judgments of the ramp stimuli.

\section{Subjects}

There were three Ss. JT, an author, is myopic, but was corrected to normal vision by a spectacle lens placed at the artificial pupil. HB and DR are emmetropic, but they used a $-1 D$ lens because it was found to increase the stability of their accommodation.

\section{Stimuli}

The test stimuli were four step stimuli, $10,16,23$, and 30 min wide, and four ramp stimuli for which $W_{1} / W_{2}=10 / 15$, $10 / 20,10 / 25$, and 10/30 min. Each stimulus was presented at three luminances: $1.8,4.5$, and $8.9 \mathrm{~mL}$. These levels were approximately $0.3,0.7$, and $1.0 \mathrm{log}$ units above threshold. Luminances near threshold were used because they provide the most sensitive test of the threshold hypothesis and because they prevent the appearance of Mach bands, which might complicate the judgment. The comparison stimuli were all step stimuli. They ranged in width from 3.3 to $40.0 \mathrm{~min}$ in steps of $3.3 \mathrm{~min}$. Their luminance was always $4.5 \mathrm{~mL}$.

\section{Procedure}

For each test stimulus at each luminance, a double-staircase procedure was used to select the comparison stimulus of the same apparent width (PSE). Test and comparison stimuli were exposed for $1 \mathrm{sec}$ each. On each trial, the test stimulus was exposed first, followed after an interval of $2 \mathrm{sec}$ by a comparison stimulus. The $\mathrm{S}$ judged the comparison wider or narrower than the test. Three seconds elapsed between trials, during which $\mathrm{E}$ changed the width of the comparison stimulus according to the double-staircase procedure (Cornsweet, 1962). This procedure was continued for 30 trials and the interpolated median of the distribution of comparison widths presented on the last 20 trials was taken as the PSE. There were 24 conditions ( 8 test stimuli by 3 luminances) and these were undertaken in random order at a rate of six conditions per day. The process was repeated until there were four measurements under each condition. Different random orders were used for each $\mathrm{S}$ and repetition.

\section{Results}

Table 1 shows the PSE for each test stimulus at each luminance. The entries are means of four repetitions. The data for each $S$ underwent separate analysis of variance. In no case was there a significant effect of luminance or a significant interaction between luminance and stimulus configuration. The threshold hypothesis requires that perceived width increase as luminance increases. Going from the lowest to the highest luminance used, the expected increases range from about $1 \mathrm{~min}$ for the $10 / 15$ stimulus to about $6 \mathrm{~min}$ for the 10/30 stimulus. Clearly, such changes did not occur.

The PSEs in Table 1 are subject to time-order error and possibly other constant errors. The errors appear to be small, i.e., the PSEs for the step stimuli are close to the actual widths. Nevertheless, the errors do complicate interpretation of the data. Therefore, an equivalent step width (ESW) was computed for each ramp stimulus. The ESW is the width of a test stimulus with step edges that matches the ramp stimulus in apparent width, i.e., has the same PSE. To this end, the linear regression of actual width upon PSE was computed for the step stimuli and the results used to obtain the ESW for each ramp stimulus. Since luminance had no effect, the PSEs were averaged over luminance levels before computation of ESW. The results are given at the bottom of Table 1. ESW values are also given for the step stimuli; the correspondence between these values and the actual widths of the step stimuli provides a rough index of the precision of the ESW values. In the case of the ramp stimuli, ESW lies about half-way between $W_{1}$ and $W_{2}$. That is, each ramp stimulus had the same apparent width as a step stimulus whose width was half-way between $W_{1}$ and $W_{2}$.

\section{EXPERIMENT 2}

Temporal forced-choice detection thresholds were obtained for the ramp stimuli and the widest and narrowest step stimuli.

\section{Subjects}

Only two Ss, DR and JT, participated in this experiment.

\section{Stimuli}

All four ramp stimuli and two step stimuli, $10 \mathrm{~min}$ and $30 \mathrm{~min}$ wide, were used. Exposure duration was $1 \mathrm{sec}$.

Table 1

PSEs and Equivalent Step Widths in Minutes of Visual Angle

\begin{tabular}{lllllll} 
& \multicolumn{3}{c}{ Step Stimuli } & & \multicolumn{2}{c}{ Ramp Stimuli } \\
$10^{\prime}$ & $16^{\prime}$ & $23^{\prime}$ & $30^{\prime}$ & $10 / 15$ & $10 / 20$ & $10 / 25$ \\
& \multicolumn{4}{c}{ PSE } & &
\end{tabular}

$10 / 30$

$\begin{array}{llllllllll}\text { DR } & 1.78 \mathrm{~mL} & 8.8 & 16.5 & 23.8 & 31.2 & 12.4 & 15.8 & 17.7 & 22.0\end{array}$

$\begin{array}{lllllllll}4.46 \mathrm{~mL} & 9.8 & 16.2 & 23.5 & 31.2 & 12.4 & 14.5 & 17.5 & 21.9\end{array}$

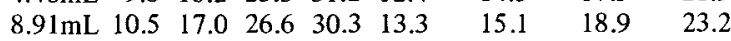

$\begin{array}{llllllllll}\text { JT } & 1.78 \mathrm{~mL} & 9.5 & 17.7 & 25.3 & 30.7 & 13.3 & 16.7 & 21.4 & 23.9\end{array}$

$\begin{array}{lllllllll}4.46 \mathrm{~mL} & 10.1 & 17.4 & 25.1 & 31.1 & 14.3 & 16.2 & 20.3 & 25.1\end{array}$

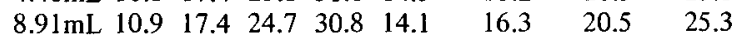

$\begin{array}{llllllllll}\text { HB } & 1.78 \mathrm{~mL} & 10.3 & 17.6 & 24.7 & 31.3 & 14.1 & 15.5 & 20.0 & 20.6\end{array}$ $\begin{array}{lllllllll}4.46 \mathrm{~mL} & 10.5 & 16.8 & 24.8 & 31.0 & 13.5 & 15.0 & 18.1 & 21.7\end{array}$

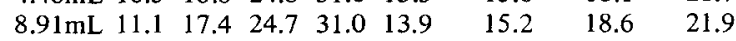

\begin{tabular}{lrrrrrrrr} 
& \multicolumn{8}{c}{ Equivalent Step-Width } \\
DR & 10.1 & 16.6 & 23.3 & 30.0 & 12.9 & 15.3 & 18.0 & 22.0 \\
JT & 9.7 & 16.8 & 24.0 & 29.6 & 13.3 & 15.7 & 19.4 & 23.4 \\
HB & 10.0 & 16.4 & 23.7 & 29.9 & 13.1 & 14.5 & 18.0 & 20.4 \\
\hline
\end{tabular}




\section{Procedure}

Each trial consisted of two test intervals, separated by a 1-sec pause. A tone identified each interval. The stimulus was exposed during one of these intervals, randomly selected, and $\mathrm{S}$ sought to identify which one. Four seconds elapsed between trials. For each stimulus, a total of 100 trials was given at each of five luminances spaced $0.1 \mathrm{log}$ units apart. The same five luminances were used for all stimuli. There were 10 sessions for each S. During each session there were 10 trials at each luminance for each of the stimuli. For each stimulus, the trials at each luminance were blocked and the luminances were presented in descending order. The order in which the stimuli were examined was varied from day to day.

The proportion of correct responses at each luminance was corrected for guessing. The corrected proportions ranged from 0.12 to 0.90 . These proportions were converted to $\mathrm{z}$-scores, a linear regression line was fitted to the five scores for each stimulus, and the 50\% threshold was computed from the equation for the line. In addition, the z-scores for each $S$ underwent two-way analysis of variance (Stimuli by Luminances).

\section{Results}

The thresholds are shown in Table 2. The analysis of variance showed the overall difference among stimuli to be significant for each $\mathrm{S}(\mathrm{DR}, \mathrm{p}<.05 ; \mathrm{JT}, \mathrm{p}<.01)$. Examination of Table 2 shows that the difference stems primarily from the low thresholds for the $30-\mathrm{min}$ step stimulus. For the most part, the thresholds for the ramp stimuli are similar to each other and to the threshold for the 10 -min step stimulus. In the case of JT, the 10/30 threshold is highly elevated, but no such elevation appears in DR's data.

The decrease in threshold between the 10-min and 30-min step stimuli illustrates the well-documented effect of area upon threshold intensity (Graham, Brown, \& Mote, 1939; Blackwell, 1946). The significant feature of the data is that there is no comparable decrease for the ramp stimuli, even though both overall width $W_{2}$ and apparent width were increased. Ramp stimuli have been found perceptually dimmer and less visible than step stimuli (Enoch, 1958; Thomas \& Kovar, 1965). Apparently, this reduction in visibility counteracted the area-intensity relationship seen in the data for the step stimuli.

\section{DISCUSSION}

The perceived width of each ramp stimulus fell about midway between the values of $W_{1}$ and $W_{2}$. It was not affected by the five-fold change in luminance which was part of Experiment 1 . This stability rules out the simple threshold hypothesis, leaving the explanations which are based on spatial interactions.

One possibility is that the apparent width represents the distance over which the sensation magnitude (von Békésy, 1960) resulting from the stimulus plus background is greater than that produced by the background alone. Because of the undershoot that lateral inhibitory interactions produce at the bottom of the edge-gradient, this distance will be less than $\mathrm{W}_{2}$, although greater than $W_{1}$. To the extent that certain requirements of linearity are met, the distance is independent of the luminance of the stimulus. Although spatial interactions are not linear in all respects, the requirements of the present situation are probably met (Thomas, 1968a). One shortcoming of this treatment is that both theoretical curves (von Békésy, 1960) and empirical measurements (Lowry \& De Palma, 1961) suggest that the perceived width should be closer to $W_{2}$ than was found in this study. In this respect, the data of Charman (1963) provide better support for the analysis.

Another possible approach assumes several receptive-field mechanisms which act in parallel, but which are differentially
Table 2

Detection Thresholds in $\mathrm{mL}$

\begin{tabular}{llcllll}
\hline & \multicolumn{3}{c}{ Step Stimuli } & \multicolumn{4}{c}{ Ramp Stimuli } \\
& $10^{\prime}$ & $30^{\prime}$ & $10 / 15$ & $10 / 20$ & $10 / 25$ & $10 / 30$ \\
DR & .468 & .371 & .478 & .489 & .468 & .446 \\
JT & .468 & .346 & .489 & .478 & .468 & .588 \\
Predicted & & & & & & \\
lower limit & - & - & .473 & .467 & .462 & .468 \\
\hline
\end{tabular}

sensitive (Thomas, 1968b; Thomas, Rourke, \& Wilder, 1968). Each mechanism is most sensitive to stimuli of a particular width. According to this approach, a step stimulus and a ramp stimulus appear equally wide if they excite the same mechanism. The selectivity of each mechanism derives from the receptive field: a central excitatory region flanked on both sides by antagonistic or inhibitory peripheral regions. Because the central and peripheral regions are antagonistic, only differential illumination of these regions-such as that provided by a stimulus of the appropriate width-excites the mechanism. The angular width of the central region, $\theta$, is assumed to vary from one mechanism to another. When the stimuli are all of equal height, the critical illumination variable for the central region is $F / \theta$, the ratio of the total flux falling within the central region, $F$, to the width of the region, $\theta$ (Thomas, Padilla, \& Rourke, in press). The response of the mechanism increases in proportion to this ratio, but at the same time the response is reduced by any light that falls in the peripheral regions. As a consequence of these properties, the response to a given stimulus varies from one receptive-field mechanism to another. In the case of a step stimulus, the primary response is by the mechanism whose central width most nearly matches the width of the stimulus. The primary response to a ramp stimulus is by a mechanism for which $\mathrm{W}_{1}<\theta<\mathrm{W}_{2}$. Thus, if the criterion of equivalence is that the primary response occurs in the same mechanism, a matching step-ramp pair will be such that the width of the step stimulus lies between $W_{1}$ and $W_{2}$ of the ramp stimulus. Furthermore, to the extent that the interactions within the receptive fields are linear, the same step-ramp pair will be equated regardless of their relative luminances.

Although the two stimuli of a ramp-step pair act upon the same mechanism, they do not act with equal effectiveness. The ramp stimulus is less effective, both because $F / \theta$ is less than for a step stimulus of the same luminance and because some light falls in the peripheral regions and causes inhibition. Thus, if the same receptive-field mechanisms also mediate detection, the threshold will be higher for a ramp stimulus than for a step stimulus of the same apparent width. This difference is found in the data of Table 2; the threshold decreases as the step stimuli increase in width, but no such decrease occurs as the ramp stimuli increase in apparent width.

By ignoring inhibitory effects, it is possible to calculate expected lower limits for the thresholds of the ramp stimuli. That is, at threshold the value of $\mathrm{F} / \theta$ must be at least as great for each ramp stimulus as for the corresponding step stimulus. Since the width of the step stimulus may be used as an estimate of $\theta$, it is a simple matter to compute the relative luminances at which $\mathrm{F} / \theta$ is the same for the two stimuli. From this information, and the threshold for the step stimulus, a lower limit can be estimated for the threshold of the ramp stimulus. The threshold for the step stimulus is obtained by interpolation in Table 2 of the data for the 10 and 30 min step stimuli. These computations have been carried out on the averaged data of DR and JT. The following expression was used for interpolation of the step stimulus thresholds (Woodworth \& Schlosberg, 1954; Pieron, 1929):

$$
\mathrm{L} \mathrm{A}^{\mathrm{m}}=\mathrm{K}
$$


in which $L$ and $A$ are the threshold luminance and area, respectively, of the step stimulus. Values for $m$ and $K$ were obtained by inserting the data for the 10-min and 30-min step stimuli. The value obtained for $\mathrm{m}$ was 0.24 .

The results are shown at the bottom of Table 2 . It should be emphasized that the expected values are lower limits and that, because of inhibitory effects, the observed thresholds are expected to be higher. This expectation is generally upheld, the $10 / 30$ threshold for DR being the only exception. Thus, not only do ramp stimuli have higher detection thresholds than step stimuli of the same apparent width, but the differences in the thresholds are generally of the magnitude required by the receptive-fieid analysis.

\section{REFERENCES}

BLACKWELL, H. R. Contrast thresholds of the human eye. Journal of the Optical Society of America, 1946, 36, 624-643.

CHARMAN, W. N. Some experiments concerning the limitations and errors in size measurement of small objects by visual microscopy. Journal of the Royal Microscopy Society, 1963, 82, $81-94$.

CORNSWEET, T. N. The staircase method in psychophysics. American Journal of Psychology, 1962, 75, 45-491.

ENOCH, J, M. Summated response of the retina to light entering different parts of the pupil. Journal of the Optical Society of America, $1958,48,392-405$.

GRAHAM, C. H., BROWN, R. H., \& MOTE, F. A. The relation of size of stimulus and intensity in the human eye. J. Intensity thresholds for white light. Journal of Experimental Psychology, 1939, 24, 555-573.

LOWRY, E, M., \& DE PALMA, J. I. Sine-wave response of the visual system. I. The Mach phenomenon. Journal of the Optical Society of America, 1961, 51, 740-746.

PIERON, H. De la sommation spatiale des impressions lumineuse au niveau de la fovea. Année Psychologique, 1929, 30, 87-105.

THOMAS, J. P. Equipment for varying the intensity of light. American Journal of Psychology, 1967, 80, 297-301.

THOMAS, J. P. Linearity of spatial integrations involving inhibitory interactions. Vision Research, 1968a, 8, 49-60.

THOMAS, J. P. Receptive-field model for visual perception. Proceedings of the 76th Annual Convention, American Psychological Association, $1968 \mathrm{~b}, 3,107-108$.

THOMAS, J. P., KOVAR, C, W. The effect of contour sharpness on perceived brightness. Vision Research, 1965, 5, 559-564.

THOMAS, J. P., PADILLA, G. J., \& ROURKE, D. L. Spatial interactions in identification and detection of compound visual stimuli. Vision Research, in press.

THOMAS, J. P., ROURKE, D. L., \& WILDER, D. G. Inhibitory effect of less intense stimuli upon the increment threshold for a narrow test line. Vision Research, 1968, 8, 537-542.

von BÉKÉSY, G. Neural inhibitory units of the eye and skin. Quantitative description of contrast phenomena. Journal of the Optical Society of America, 1960, 50, 1060-1070.

WOODWORTH, R, S., \& SCHLOSBERG, H. Experimental psychology. New York: Holt, Rinehart, \& Winston, 1954.

\section{NOTES}

1. This research was supported in part by United States Public Health Service Research Grant NB 07249.

2. Address: Department of Psychology, University of California, Berkeley, Calif. 94720.

(Accepted for publication December 23, 1968.) 